objective of the proposed study is to assess the effect of administering a prebiotic, such as HAMS-AB, on the gut microbiome profile, SCFA production, glycemia and $\beta$-cell function in humans with T1D. METHODS/STUDY POPULATION: We hypothesize that administration of HAMS-AB will (i) improve the gut microbiome profile in humans with T1D, (ii) increase SCFA production, and (iii) improve $\beta$-cell health, $\beta$-cell function and overall glycemia. We propose a pilot randomized controlled cross-over trial of HAMS-AB in 12 youth with newly-diagnosed T1D. We will use state-of-the-art markers to profile the gut microbiome (using 16S rRNA sequencing), measure stool SCFA levels (using gas chromatography), asses $\beta$-cell stress/death (by measuring proinsulin to C-peptide ratios) and glycemia (assessed by continuous glucose monitoring and $\mathrm{HbAlc}$ measurements). RESULTS/ANTICIPATED RESULTS: We expect that the use of HAMS-AB in newly diagnosed youth with type 1 diabetes will alter the gut microbiome profile (thus increasing the number of fermenters and SCFA levels), $\beta$-cell function and glycemia in humans with T1D. DISCUSSION/SIGNIFICANCE OF IMPACT: Given the unknown long-term effects of immune-modulatory therapy on those at risk for or those diagnosed with $\mathrm{T} 1 \mathrm{D}$, the use of a prebiotic such as HAMS-AB offers a simple, safe, yet inexpensive and tolerated dietary alternative approach to mitigating disease.

4069

Examining the Effects of A Hybrid Communication
Coaching Intervention on Fathers' Responsive
Strategy Use with Children with Autism Spectrum
Disorder
Michelle Flippin
'University of Rhode Island

OBJECTIVES/GOALS: This investigation aimed to mitigate barriers to father involvement in communication intervention for children with ASD and contribute to clinical practice by examining the effects of a hybrid parent coaching intervention for fathers of children with ASD that is tailored to fit both father's interaction and communication styles as well as individual child characteristics. The Hybrid Father Communication Coaching (HFCC) combined online parent coaching lessons with in-person father-child aquatics sessions in order to increase father's use of responsive verbal and play strategies. Distal effects on child communication were also investigated. METHODS/STUDY POPULATION: A single subject, multiple baselines across strategies experiment was conducted with one dyad (i.e., father, child with ASD). In the present study, a hybrid father coaching model was used. Parent communication coaching sessions were delivered online, and weekly, father-child aquatics sessions were conducted in person, to provide opportunities for the father to use three targeted responsive strategies (i.e., follow-in comments, follow-in directives, responsive object play) during father-child physical activity. Collateral measures of child communication skills were also investigated. Single subject designs are particularly suitable for autism interventions, as they allow for experimental control with participants who are from heterogeneous populations (McReynolds and Kearn, 1983). The child participant was 5 years, 6 months at the start of intervention and had previously received a community diagnosis of ASD. Throughout the duration of the study, the child attended full-time kindergarten and received in-school speech-language therapy, as a well as 18-20 hours per week of Applied Behavioral Analysis intervention, occupational therapy, physical therapy and speech-language therapy after school. The participating father was a biological parent who resided with the child continuously since birth. The participating father had no other formal parent training in communication intervention before participating. RESULTS/ANTICIPATED RESULTS: The hybrid father communication coaching program (HFCC) yielded positive results for both father and child participant. The father quickly achieved a high level of competency using two of the three, targeted strategies (i.e., follow-in comments, follow-in directives). However, use of a third strategy (i.e., responsive object play) was not maintained above baseline levels. The follow-in comments strategy was used by the participating father more frequently than the follow-in directives strategy. Small increases were documented for child use of spontaneous single words across intervention phases and increased single word use over was maintained eight weeks following intervention. DISCUSSION/SIGNIFICANCE OF IMPACT: The present study provided information regarding the efficacy of a clinically relevant hybrid parent-coaching program, tailored to both father and child characteristics, to enhance fathers' use of responsive strategies and increase communication skills for children with ASD.

4367

\section{Exploratory evaluation of an online educational intervention for JUUL use}

Eleanor L S Leavens ${ }^{1}$, Matthew J. Carpenter, Tracy T. Smith, and Nikki Nollen

${ }^{1}$ University of Kansas Frontiers

OBJECTIVES/GOALS: Initiation of JUUL use by young adults is one of the most significant issues of concern within the debate on vaping. Despite the proliferation of products and the surge in prevalence, no studies have investigated individual-level interventions or prevention strategies for pod-mod use. METHODS/STUDY POPULATION: Participants $(N=947)$ were young adults $(<30$ years old) recruited from Amazon's Mechanical Turk based on smoking (never, former, and current smokers) and JUUL use status (never and current users), resulting in 6 use groups. In a pre-post design, participants completed baseline assessments, were presented with a brief JUUL-specific educational intervention, and completed post-assessment measures. The one-page intervention provided basic information about JUUL and stated that JUUL is harmful to non-smokers but could be beneficial to smokers if they completely switch. Primary outcomes were changes in JUUL knowledge, perceived harmfulness, intentions for future use, and motivation to change. RESULTS/ANTICIPATED RESULTS: Participants $\left(M_{\text {age }}=26.1\right)$ were male $(57 \%)$ and White $(75 \%)$. Overall, the intervention increased JUUL-related knowledge, risk perceptions, commitment to quitting, and readiness to quit JUUL $(p s<.01)$. Similarly, participants showed decreased interest in future JUUL use, interest in purchasing JUUL, and interest in future regular use $(p s<.01)$. Non-JUUL users showed decreased interest in initiating JUUL use after viewing the intervention $(p<.01)$. There were significant Time X Group interactions for JUUL-related knowledge $(p<.001)$, with never JUUL/never smokers showing the greatest increase in product knowledge following the intervention. However, no other interaction effects were significant. DISCUSSION/SIGNIFICANCE OF IMPACT: The intervention was effective in increasing knowledge and risk perceptions while reducing intentions for future use. The intervention was most effective in increasing knowledge among non-users, suggesting that brief educational interventions may be useful tools for preventing podmod initiation. CONFLICT OF INTEREST DESCRIPTION: Dr. 